PAPER

\title{
Giant axonal neuropathy: clinical and genetic study in six cases
}

\author{
E Demir, P Bomont, S Erdem, L Cavalier, M Demirci, G Kose, S Muftuoglu, A N Cakar, E Tan, \\ S Aysun, M Topcu, P Guicheney, M Koenig, H Topaloglu
}

J Neurol Neurosurg Psychiatry 2005;76:825-832. doi: 10.1136/jnnp.2003.035162

See end of article for
authors' affiliations
.....................
Correspondence to:
Dr H Topaloglu, Hacettepe
Cocuk Hastahanesi, Cocuk
Noroloji Bolumu, 06100,
Ankara, Turkey;
htopalog@hacettepe.edu.tr
Received
29 December 2003
Revised version received
28 July 2004
Accepted
22 September 2004

Background: Giant axonal neuropathy (GAN) is a severe recessive disorder characterised by variable combination of progressive sensory motor neuropathy, central nervous system (CNS) involvement, and "frizzly" hair. The disease is caused by GAN gene mutations on chromosome 16q24.1.

Aims: To search for GAN gene mutations in Turkish patients with GAN and characterise the phenotype associated with them.

Methods: Linkage and mutation analyses were performed in six affected patients from three consanguineous families. These patients were also investigated by cranial magnetic resonance imaging (MRI) and electroencephalography (EEG). Electromyography (EMG) was performed in heterozygous carriers from family 1 and family 3 .

Results: Linkage to 16q24.1 was confirmed by haplotype analysis. GAN mutations were identified in all families. Family 1 had the R293X mutation, previously reported in another Turkish family. Families 2 and 3 , originating from close geographical areas, shared a novel mutation, 1502+1G $>T$, at the donor splice site of exon 9. All patients displayed a common phenotype, including peripheral neuropathy, cerebellar ataxia, and frizzly hair. Cranial MRI showed diffuse white matter abnormalities in two patients from family 1 and the patient from family 3 , and minimal white matter involvement in the patient from family 2 . EMG of a heterozygous R293X mutation carrier showed signs of mild axonal neuropathy, whereas a 1502+1G > T mutation carrier had normal EMG. EEG abnormalities were found in three patients.

Conclusion: These findings highlight the association of CNS involvement, in particular white matter abnormalities, with peripheral neuropathy in GAN. The phenotypical consequences of both mutations (when homozygous) were similar.

G iant axonal neuropathy (GAN; MIM 256850) is a recessive neurodegenerative disorder affecting both the peripheral nervous system and the central nervous system (CNS). ${ }^{1-4}$ Neurological abnormalities are usually associated with a characteristic "frizzly" hair abnormality, although several patients with normal hair structure have been reported.$^{5-7}$ A Tunisian form of GAN with normal hair, slower progression, and multisystem degeneration including motor neurone degeneration was also described. ${ }^{8}$ Electron microscopic examination of axonal swellings in peripheral nerves revealed excessive accumulation of neurofilaments in GAN. An aggregation of cytoplasmic intermediate filaments was also seen in other cell types, including fibroblasts, endothelial cells, melanocytes, and Langerhans cells, indicating a generalised disorganisation of cytoplasmic intermediate filaments in GAN. ${ }^{10}$

We and others have located the GAN disease locus to 16q24.1 by homozygosity mapping in consanguineous families, ${ }^{11-13}$ and we have recently identified the defective gene by positional cloning. ${ }^{14}$ We found one single nucleotide insertion, nine missense, and four nonsense mutations, distributed throughout the 11 exons of the GAN gene, in 12 families of various origins. Subsequently, additional homozygous or compound heterozygous mutations of the GAN gene have been reported in other patients with GAN. ${ }^{15-18}$ The defective protein, named gigaxonin, contains an $\mathrm{N}$-terminal $\mathrm{BTB} / \mathrm{POZ}$ domain and C-terminal kelch repeat domains, which are possibly implicated in protein-protein interactions. Gigaxonin, whose function is not known exactly, is distantly related to other $\mathrm{BTB} / \mathrm{kelch}$ proteins, several of which are implicated in the actin cytoskeletal network. ${ }^{19-21}$ Physiologically, it colocalises with microtubule associated protein 1B (MAP1B) in neurones. ${ }^{22}$ Gigaxonin binds directly to microtubule associated protein light chain (MAPIB-LC), ${ }^{22}$ which is involved in microtubule stability and actin filament binding. ${ }^{23}$ The interaction of gigaxonin with MAPIB-LC enhances the microtubule stability required for axonal transport. ${ }^{22}$ Moreover, microtubule destabilisation has been shown to induce intermediate filament aggregation in GAN fibroblasts, suggesting a role for gigaxonin in intermediate filament and microtubule networks. ${ }^{24}$ Here, we present clinical and genetic analyses of six Turkish patients from three consanguineous families with GAN.

\section{SUBJECTS}

Three consanguineous families were investigated in our study (fig 1). All families gave informed consent. The clinical diagnosis of GAN was made on the following criteria: progressive sensory motor peripheral neuropathy; features of CNS involvement such as mental retardation, cerebellar abnormalities, pyramidal tract signs, and cranial nerve abnormalities; frizzly hair; electromyography (EMG) findings of axonal neuropathy; and ultrastructural findings of increased cytoplasmic intermediate filaments in sural nerve or skin specimens. A sural nerve biopsy from the index patient in family 1 (patient 1 ) and skin biopsies from patient 2 in family 1 and patient 5 in family 2 were obtained. No biopsy sample was available from patient 6 in family 3. The diagnosis of GAN was

Abbreviations: $E E G$, electroencephalography; EMG, electromyography; GAN, giant axonal neuropathy; MAP1B, microtubule associated protein 1B; MAPIB-LC, microtubule associated protein IB light chain; MNCV, motor nerve conduction velocity; MRI, magnetic resonance imaging; PCR, polymerase chain reactions; SSCP, single strand conformational polymorphism 

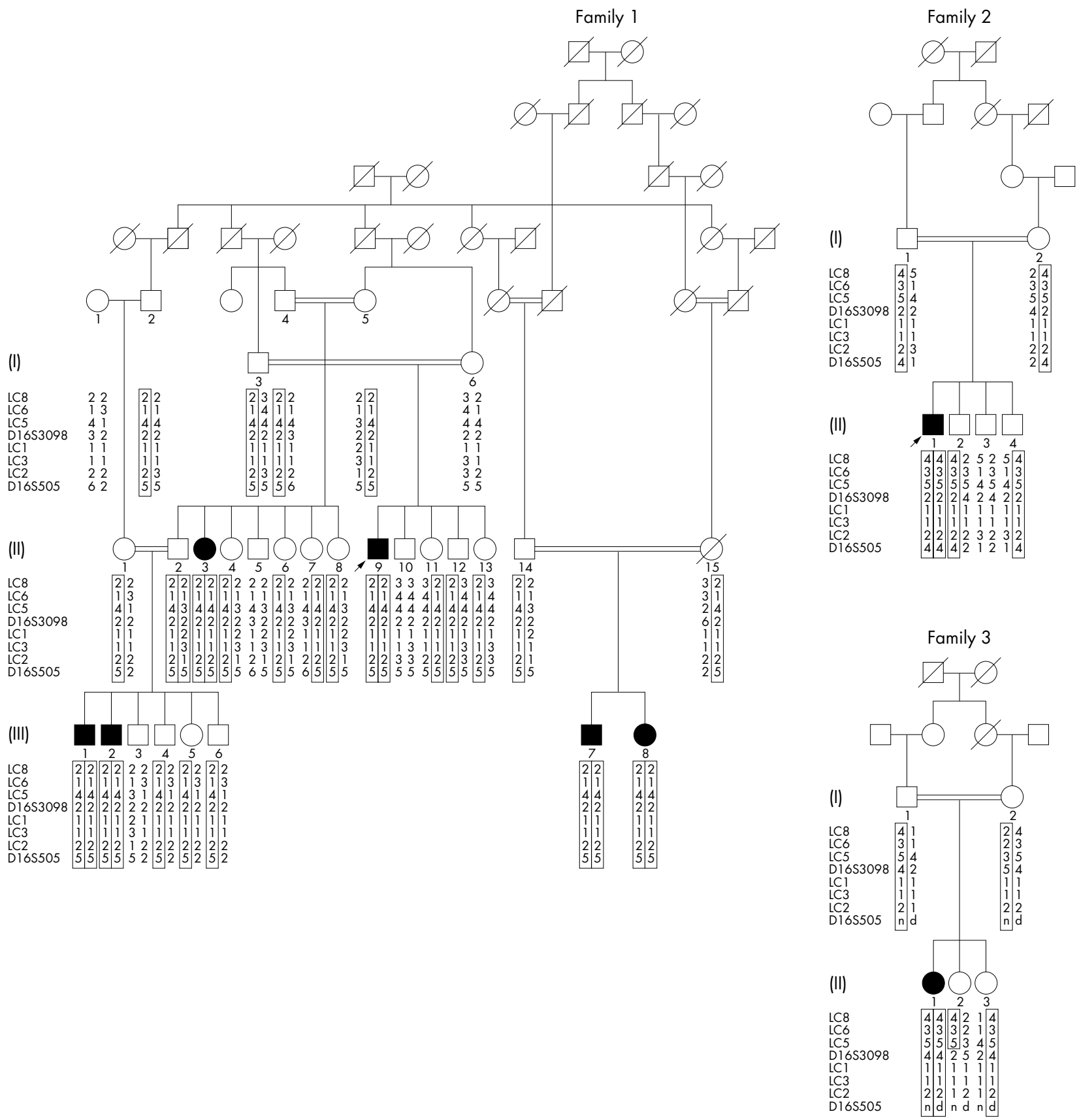

Figure 1 Pedigree of three families with giant axonal neuropathy showing linkage to chromosome 16q24.1. Affected individuals are represented by closed symbols and unaffected individuals are represented by open symbols. Disease bearing haplotypes are boxed for each family; nd, not determined.

based solely on clinical grounds for this patient. Direct physical examination of two affected siblings in family 1 (individuals (III)- 1 and (III)-2) was not possible, but they were reported to have distal limb weakness, truncal ataxia, mental deterioration, frizzly hair, and skeletal deformities, resembling phenotypically the other patients in the same family. Heterozygous carriers displayed none of the clinical features of GAN and their physical examination was normal. All families originated from southeastern Turkey.

\section{METHODS}

\section{Electrophysiological and neuroimaging studies}

EMG and electroencephalography (EEG) were performed in four patients and six patients, respectively. EMG was also carried out in two clinically unaffected heterozygous carriers (individual (II)-14 in family $\mathrm{l}$ and individual (I)- 1 in family 3). Other heterozygous carriers did not consent to EMG. Patients 1, 2, 5, and 6 underwent cranial magnetic resonance imaging (MRI). MRI examinations were performed on $1.5 \mathrm{~T}$ systems. Cranial MRI examinations included $\mathrm{Tl}$ and $\mathrm{T} 2$ weighted axial and coronal images.

\section{Histological and ultrastructural analyses Nerve biopsy}

Sural nerve samples were obtained from $5-6 \mathrm{~cm}$ above the lateral malleolis. One part of the nerve was snap frozen in liquid nitrogen and $12 \mu \mathrm{m}$ frozen sections were stained with haematoxylin and eosin and modified Gomori trichrome. The 
rest of the specimen was fixed in 3\% glutaraldehyde, postfixed in $1 \%$ osmium tetroxide, dehydrated through serial alcohol baths, and embedded in Spurr's resin. Semithin sections for light microscopy were stained with toluidine blue. Ultrathin sections were contrasted with uranylacetate and lead citrate and examined under a Zeiss 952 electron microscope.

\section{Skin biopsy}

Skin specimens were fixed in $2.5 \%$ phosphate buffered glutaraldehyde, washed in the same buffer, and then postfixed in $1 \% \mathrm{OsO}_{4}$. After dehydration in increasing concentrations of ethanol, the specimens were embedded in araldite (CY 212). Semithin sections were stained with toluidine blue. Ultrathin sections were contrasted with uranylacetate and lead citrate and examined under a Zeiss 952 electron microscope.

\section{Genetic analysis}

Microsatellite marker analysis

Samples of venous blood were obtained from patients and relatives after informed consent. The three families affected by GAN were genotyped with polymorphic markers present in the GAN region on chromosome 16q24.1 (LC8, LC6, LC5, D16S3098, LC1, LC3, LC2, and D16S505). ${ }^{13}$ Forward primers were labelled with 6-Fam, Hex, or Ned fluorochromes (Applied Biosystems Inc, Foster City, California, USA). Polymerase chain reactions (PCRs) were performed in a final volume of $10 \mu \mathrm{l}$ containing $20 \mathrm{ng}$ of genomic DNA, ImM dNTP, $1 \times$ NBL buffer, $0.50 \mu \mathrm{M}$ of each primer, and $0.1 \mathrm{IU}$ of Taq polymerase. The fluorescent PCR products were separated and detected on $4.25 \%$ acrylamide gels with an ABI 377 DNA sequencer, and alleles were assigned with the Genotyper software (v2.5; Applied Biosystems).

\section{Mutation analysis}

The 11 exons of the GAN gene were screened for mutations at the genomic level. The sequence of the primers has been published (http://genetics.nature.com/supplementary info/). ${ }^{14}$ PCR products were analysed by single strand conformational polymorphism (SSCP) on PlusOne precast acrylamide gels (Pharmacia, Uppsala, Sweden) with a Genephor electrophoresis device (Pharmacia) at three different running temperatures $\left(10^{\circ} \mathrm{C}, 15^{\circ} \mathrm{C}\right.$, and $\left.20^{\circ} \mathrm{C}\right)$ and silver stained. Electrophoretic variants were sequenced from both the forward and reverse strands.

\section{RESULTS}

\section{Clinical features}

Table 1 outlines the clinical features of the six patients investigated. All patients had a similar clinical presentation, compatible with a degenerative disease. They were born after an uneventful pregnancy and delivery. Psychomotor development was normal in these patients. Onset of disease varied from 3.5 to 4.5 years of age. Distal limb weakness was the initial complaint in all patients. In addition, truncal ataxia contributing to unsteady gait was noticed at the beginning of the disease in two patients (patients 3 and 6). Cerebellar abnormalities became apparent within a few years in other patients. Mental deterioration was noted to start before the age of 10 years. All patients showed a progressive clinical course. Patients 1, 4, 5, and 6 became wheelchair bound around 9-10 years of age. None of the patients had seizures or seizure-like episodes.

\section{Physical examination}

All patients were of short stature, being under the third centile. They had pale and curled hair, known as frizzly hair, and dry skin. A peculiar facial appearance characterised by facial diplegia, ptosis, and a prominent high forehead was present. Two female patients (patients 4 and 6) had early breast development consistent with puberte precocious. Boys showed no signs of early pubertal development. Scoliosis was present in five patients (table 1). Other skeletal deformities consisting of various combinations of pectus carinatum, genum-valgus, pes equino-valgus, and pes planus were also noted in these five patients.

\section{Neurological examination}

All patients had mild mental retardation except for the youngest one (patient 3). Optic fundus examinations were normal. Five patients had slight facial weakness characterised by flattening of the nasolabial sulci and inability to raise the eyebrow. Ptosis was present in four patients. Most patients showed generalised hypotonia, which clearly predominated in the distal lower limbs. Pronounced distal muscle weakness and atrophy were present in the lower limbs. The power of the distal limb muscles ranged between 2 of 5 and $3+$ of 5 . Ankle jerks were absent. Weakness was less pronounced in the upper extremities. Patients 4, 5, and 6 presented moderate to severe upper limb weakness, with tenar-hypotenar atrophy of the hands, whereas milder muscle weakness with normal or hypoactive deep tendon reflexes was present in the other patients. Mild impairment of pain and light touch sensations was observed. All patients had truncal ataxia, nystagmus, dysmetria, and dysarthria. Plantar responses were extensor. There was no sign of spasticity.

\section{Electroencephalography}

Bilateral synchronous sharp wave discharges, most prominent in the left anterior regions, were seen in the EEG of patient 1 . The EEG of patient 4 showed spike-slow wave discharges in central areas. Sharp wave and sharp-slow wave discharges in the right centroparietal and parietooccipital regions with spreading to homologous regions of the left hemisphere were seen in the EEG of patient 5. The EEGs of patients 2,3, and 6 were normal.

\section{Electromyography}

The EMGs of patients 1, 2, 5, and 6 were compatible with sensory motor neuropathy of axonal type. Sensory action potentials were absent or significantly decreased. Motor nerve conduction velocity (MNCV) was slowed to the demyelinating range in the lower limbs of patients 2 and 5 (table 1). Patient 1 had normal MNCV values in the lower limbs. MNCV was normal or mildly slowed in the upper limbs of all patients. An EMG of the heterozygous carrier in family 1 (individual (II)-14) at age 41 years showed bilateral mild to moderately reduced compound nerve action potential amplitudes of the sural nerves, which was compatible with mild axonal neuropathy (left sural sensory nerve action potential, $2.1 \mu \mathrm{V}$; right sural sensory nerve action potential, $2.4 \mu \mathrm{V}$; normal value, $>5 \mu \mathrm{V})$. MNCV was normal in this individual. The heterozygous carrier in family 3 (individual (I)-1) had a normal EMG.

\section{Magnetic resonance imaging}

Table 2 outlines the MRI findings in four patients. MRI scans were available for patients 1, 2, 5, and 6 at ages $11,10,5$, and 16 years, respectively. The MRI scans showed diffusely increased $\mathrm{T} 2$ and decreased $\mathrm{T} 1$ signal intensities in the anterior and posterior periventricular and cerebellar white matter of patients 1, 2, and 6, but minimal hyperintensity in the cerebellar white matter and no change in the periventricular white matter of patient 5 (figs 2 and 3). Subcortical white matter was spared in these patients except for patient 1, who had slight involvement of subcortical U fibrils. The 
Table 1 Clinical data of the patients

\begin{tabular}{|c|c|c|c|c|c|c|}
\hline Patients & 1 & 2 & 3 & 4 & 5 & 6 \\
\hline Family/number & $1 /(I I)-9$ & $1 /(I I I)-7$ & $1 /(I I I)-8$ & $1 /(I I)-3$ & $2 /(I I)-1$ & $3 /(I I)-1$ \\
\hline Age/sex & $13 \mathrm{y} / \mathrm{M}$ & $12 \mathrm{y} / \mathrm{M}$ & $5 \mathrm{y} / \mathrm{F}$ & $13.5 \mathrm{y} / \mathrm{F}$ & $11 \mathrm{y} / \mathrm{M}$ & $16 \mathrm{y} / \mathrm{F}$ \\
\hline Age at onset & $4 y$ & $3.5 y$ & $4 y$ & $4.5 y$ & $4 y$ & $4.5 y$ \\
\hline Frizzly hair & Yes & Yes & Yes & Yes & Yes & Yes \\
\hline Scoliosis & Yes & Yes & No & Yes & Yes & Yes \\
\hline Precocious puberty & No & No & No & Yes & No & Yes \\
\hline Mental deterioration & + & + & No & + & + & + \\
\hline Facial weakness & ++ & + & No & ++ & ++ & + \\
\hline Ptosis & ++ & + & No & + & ++ & No \\
\hline Cerebellar signs & Yes & Yes & Yes & Yes & Yes & Yes \\
\hline Babinski's sign & Yes & Yes & Yes & Yes & Yes & Yes \\
\hline Peripheral neuropathy & Yes & Yes & Yes & Yes & Yes & Yes \\
\hline MNCV (median nerve) & $47 \mathrm{~m} / \mathrm{sec}$ & $39 \mathrm{~m} / \mathrm{sec}$ & ND & ND & $43 \mathrm{~m} / \mathrm{sec}$ & $37.6 \mathrm{~m} / \mathrm{sec}$ \\
\hline MNCV (peroneal nerve) & $44 \mathrm{~m} / \mathrm{sec}$ & $22 \mathrm{~m} / \mathrm{sec}$ & ND & ND & $28 \mathrm{~m} / \mathrm{sec}$ & Undetectable \\
\hline EEG abnormality & Sharp wave & Normal & Normal & Spike-slow wave & Sharp wave Sharp-slow wave & Normal \\
\hline Motor capacity & WCB & Walking with aid & Walking & WCB & WCB & WCB \\
\hline
\end{tabular}

EEG, electroencephalography; $F$, female; $M$, male; $M N C V$, motor nerve conduction velocity; $N D$, not done; WCB, wheel chair bound; $y$, years; +, mild; ++, moderate.

posterior crus of the internal capsule showed increased signal intensity on T2 weighted images in cranial MRI scans of patients 1,2 , and 6 . Increased T2 weighted signal intensities were present in the posteromedial thalami of patient 6 . Atrophy of the cerebellum and cervical spinal cord and increased T2 weighted signal intensity of the brainstem were evident in patients 1 and 6 . Two patients received contrast material, and contrast enhancement in parietal white matter and cerebellum was seen in the MRI scans of patients 2 and 6 , respectively. The cavum septi pellucidi et vergae abnormality was present in all patients. The corpus callosum and basal ganglia were normal in all of the patients.

\section{Light microscopy}

Light microscopy of the sural nerve from patient 1 showed a moderate reduction in the number of myelinated fibres (fig 4). Several fibres with distended axons (up to 20-25 $\mu \mathrm{m}$
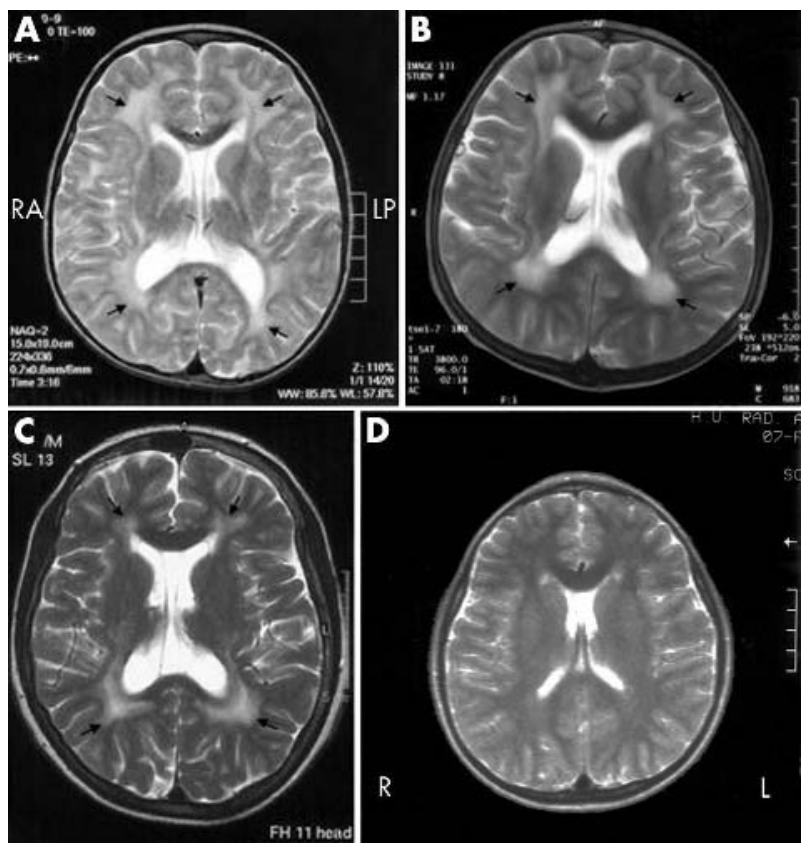

Figure 2 (A-C) T2 weighted axial magnetic resonance imaging (MRI) of the brain in patients 1, 2, and 6, respectively, showing increased signal intensity in the anterior and posterior periventricular white matter (arrows). (D) T2 weighted axial MRI of the brain in patient 5 showing no signal change in the periventricular white matter. in diameter) and thin myelin sheaths were noted among normal appearing myelinated fibres.

\section{Electron microscopy}

Nerve biopsy

Electron microscopy examination of the sural nerve from patient 1 showed both normal and giant sized axons. The axoplasm of axonal swellings was completely filled with tightly packed neurofilaments (fig 5A). Myelin sheets surrounding the swollen axons were abnormally thin.

\section{Skin biopsy}

The cytoplasm of fibroblasts from patients 2 and 5 showed an accumulation of intermediate filaments forming whorls (fig 5B).
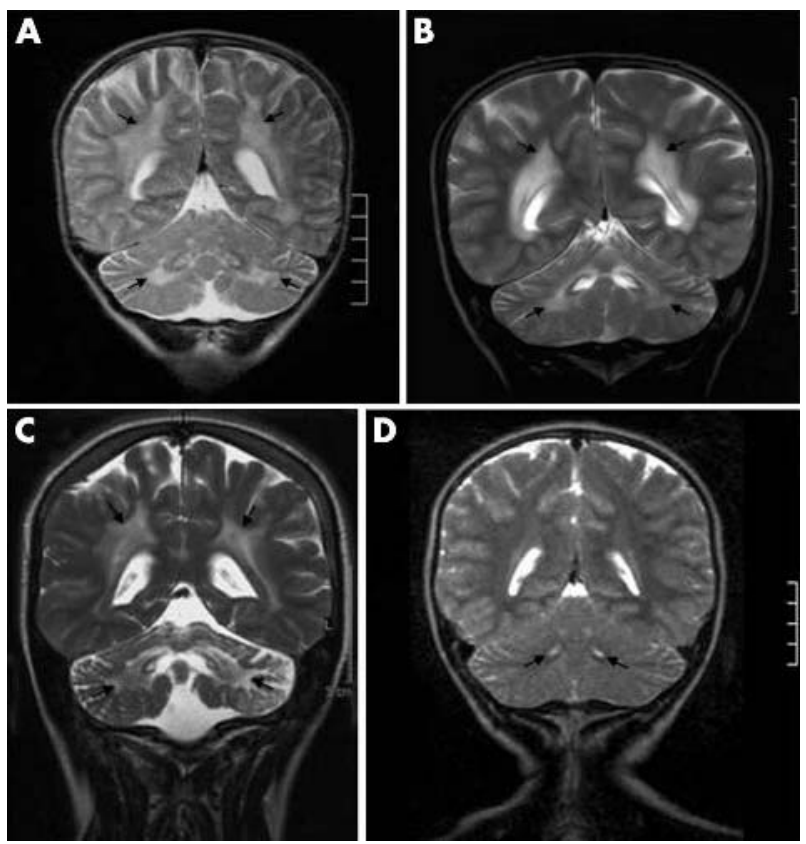

Figure 3 (A-C) T2 weighted coronal magnetic resonance imaging (MRI) of the brain in patients 1,2, and 6, respectively, showing significantly increased signal intensity in the periventricular and cerebellar white matter (arrows). (D) T2 weighted coronal MRI of the brain in patient 5 showing minimally increased signal intensity in the cerebellum (arrows). 
Table 2 Magnetic resonance imaging findings in the patients

\begin{tabular}{lllll}
\hline & Patient 1 & Patient 2 & Patient 5 & Patient 6 \\
\hline Age at imaging (years) & 11 & 10 & 5 & 16 \\
White matter abnormality & Anterior-posterior & Anterior-posterior & Minimal & Anterior-posterior \\
Subcortical white matter abnormality & PV, C & PV, C & C & PV, C \\
Brainstem involvement & + & - & - & - \\
Cerebellar atrophy & + & - & - & + \\
Posterior crus of the internal capsule & + & - & - & + \\
Thalamic involvement & - & + & - & + \\
Cavum septi pellucidi et vergae & + & - & - & + \\
Basal ganglia abnormality & - & + & + & + \\
\hline C, cerebellar; PV, periventricular. & & & - & - \\
\end{tabular}

\section{Other laboratory investigations}

The index patient of family 1 (patient 1) and the patients from families 2 and 3 had normal blood glucose, renal and liver function, vitamin B12, and arylsulfatase enzyme activity. Vitamin B12, vitamin E, thyroid hormones, and the blood glucose concentration were also normal in the heterozygous carrier from family 1 (individual (II)-14), who had subclinical neuropathy.

\section{Genetics}

Haplotype analysis

All patients from the three consanguineous families were homozygous for at least seven consecutive markers encompassing the GAN locus, and the healthy siblings had different genotypes (fig 1). In addition, all six affected members from family 1 were homozygous for the same haplotype. The results confirm linkage to $16 q 24.1$ for all three families.

The patients in families 2 and 3, originating from the same region of Turkey, shared a common allele for six of the seven closely linked polymorphic markers tested. However, alleles at marker D16S3098, which is located immediately distal to GAN, differed by two CA repeats between the two families (fig 1).

\section{Mutation analysis}

Individuals (III)- 1 and (III)-7 in family 1 were studied by SSCP for the 11 exons of the GAN gene. The electrophoretic variants were sequenced, allowing identification of the same homozygous mutation for each branch of the family: a $\mathrm{C}$ to $\mathrm{T}$ transition at position 877 on the CDNA, resulting in the occurrence of a stop codon at amino acid position 293 (R293X) (fig 6). We identified a novel homozygous mutation in the affected individuals in families 2 and $3:$ a $G$ to $T$

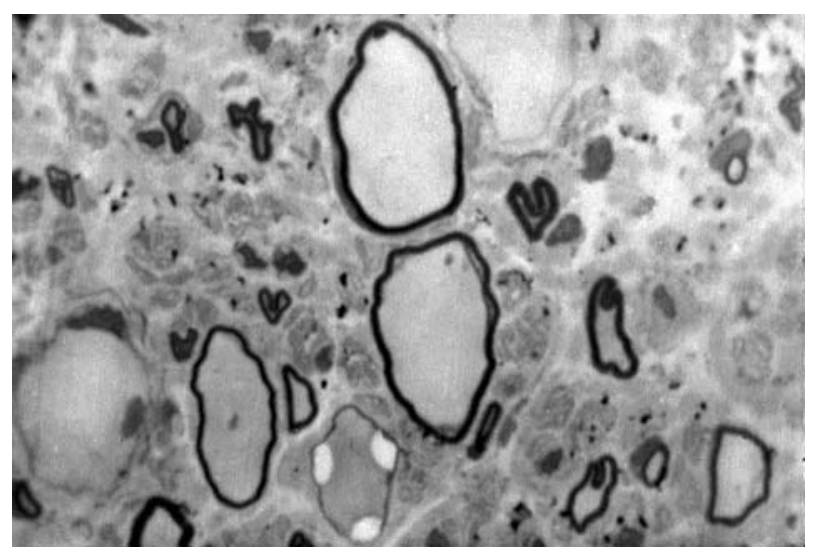

Figure 4 Semithin section of the sural nerve from patient 1 showing several giant axons with thin myelin sheath (original magnification, $\times 1428)$.
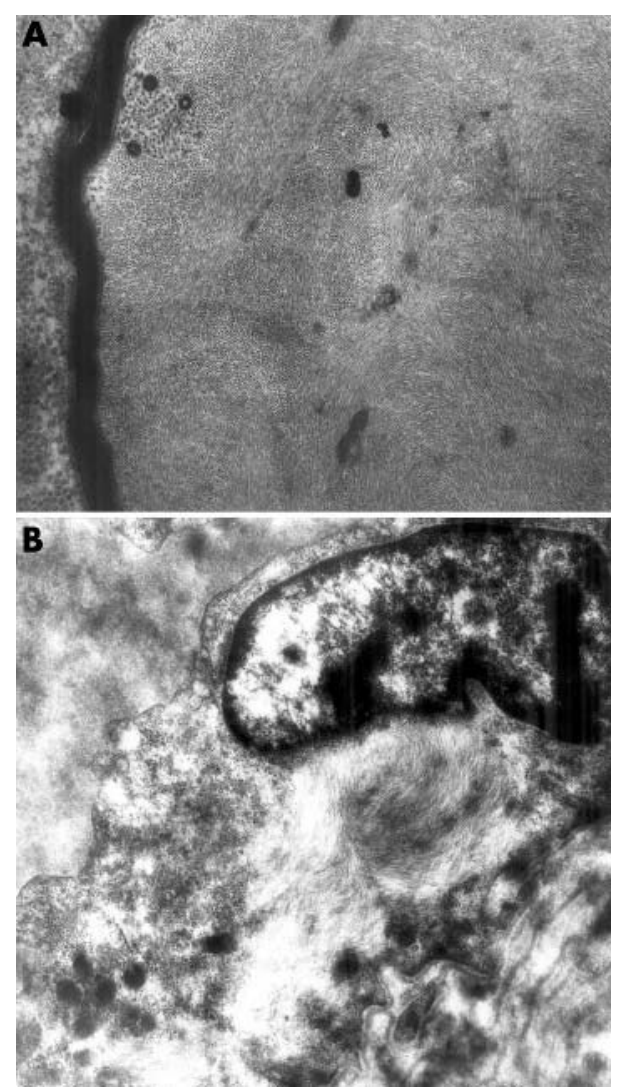

Figure 5 (A) Electron microscopy of the sural nerve from patient 1 showing a giant axon filled with masses of neurofilaments (original magnification, $\times 152500$ ). (B) Accumulation of whorly intermediate filaments in fibroblasts from patient 5 (original magnification, $\times 77500$ ).

transversion at position $1502+1$, which affects the donor splice site of exon 9 (fig 6). The $1502+1 \mathrm{G}>\mathrm{T}$ mutation was not identified in the 100 chromosomes from Turkish control subjects by direct sequencing.

\section{DISCUSSION}

\section{Clinical features}

All patients showed a homogeneous clinical picture, mainly characterised by the involvement of neuroectodermal systems, including peripheral neuropathy, CNS features, and frizzly hair. Peripheral neuropathy dominated the clinical picture at the beginning of the disease. Distal limb weakness caused by peripheral neuropathy was the presenting feature. Progressive sensory motor neuropathy was found to be primarily axonal. Secondary demyelination may also be associated with axonal degeneration in GAN..$^{25}$ We found 

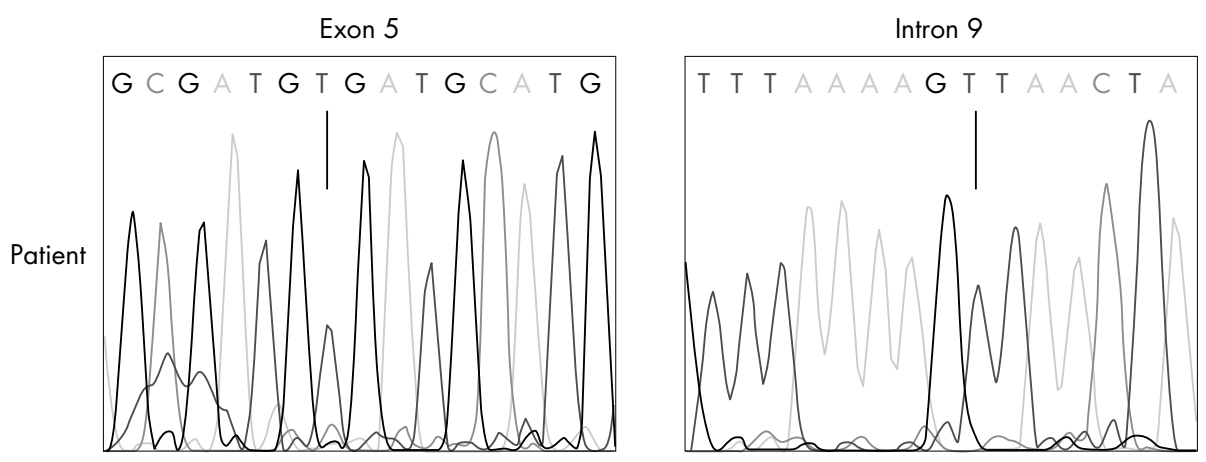

Figure 6 Electropherogram profiles of mutations $877 \mathrm{C}>\mathrm{T}$ in family 1 and $1502+1 \mathrm{G}>\mathrm{T}$ in families 2 and 3 .
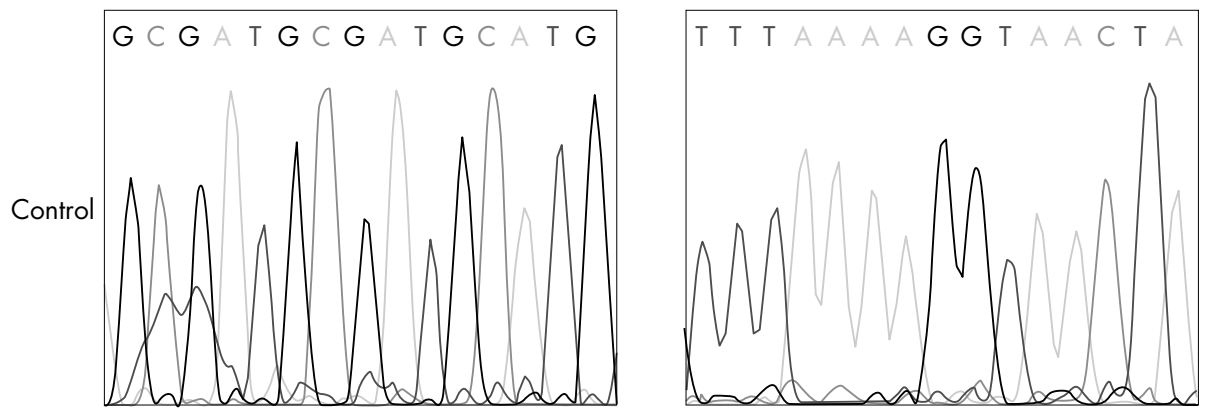

moderate to severely decreased motor nerve conduction velocities, presumably as a result of demyelination, in two of the four patients studied by EMG.

A generalised involvement of CNS structures such as the cerebral cortex, cerebellum, brainstem, and pyramidal tracts was shown in postmortem studies of patients with GAN. ${ }^{27} 28$ Cerebellar dysfunction was the most common CNS manifestation in our patients. It became symptomatic earlier than the other CNS symptoms. Cerebellar ataxia accompanied distal limb weakness at initial presentation in two patients. Cranial nerve impairment was restricted to facial and oculomotor nerves, causing facial weakness and ptosis, respectively. However, a more diffuse dysfunction of the brainstem, with involvement of multiple cranial nerves, was also described in patients with GAN. ${ }^{39}{ }^{30}$ Mental deterioration started in a later phase of the clinical course and progressed slowly.

Puberte precocious is an unusual and rare clinical feature of GAN. Two female patients had early breast development according to their chronological age. It has been suggested that this feature is caused by CNS involvement. ${ }^{31}{ }^{32}$ However, no sign of puberte precocious was present in the male patients at similar ages.

Our patients had a severe clinical phenotype, which presented in a stereotypical fashion as outlined above, in contrast to some unusual presentations of GAN in the literature. Predominant expression of CNS involvement, including epileptic seizures and mental deterioration, was seen in a patient with GAN and mild sensory neuropathy. ${ }^{33}$ In contrast, mild CNS involvement has been reported to occur many years after the onset of peripheral neuropathy in some patients with GAN. ${ }^{634}$

\section{EEG features}

Electrophysiological evidence of CNS involvement was present in three patients. Disorganised background activity with focal spikes, spike-slow wave discharges, and paroxysmal slow wave activity were reported in GAN. ${ }^{10} 3133$ None of our patients had seizures, but focal or generalised EEG abnormalities characterised by sharp wave paroxysms, sharpslow wave, and spike-slow wave discharges were seen in three patients (table 1). These discharges showed no particular distribution, originating from centroparietal, anterior, or posterior regions, sometimes with secondary generalisation.

\section{MRI features}

Neuroimaging findings described in GAN include widespread white matter demyelination and atrophy of the cerebellum, brainstem, spinal cord, and corpus callosum. ${ }^{24} 33$ 35-37 White matter abnormality was the most common MRI finding in our patients. In patients 1,2 , and 6 , diffuse white matter lesions were present in the anterior and posterior periventricular regions and cerebellum, extending into the subcortical white matter. However, cranial MRI of patient 5 showed limited involvement of the cerebellar white matter, perhaps because MRI was carried out at an earlier age in this patient. Progression of white matter lesions has been shown in patients with GAN. ${ }^{35}$ White matter involvement of GAN does not establish a specific pattern. Frontoparietal and periventricular white matter are affected more prominently in some patients with GAN, even sometimes sparing the cerebellum. ${ }^{35}{ }^{36}$ In contrast, more pronounced involvement of the cerebellar and occipital white matter compared with the frontal regions was also seen in GAN. ${ }^{33}{ }^{37}$ Demyelination with gliotic changes, few giant axons, and numerous astrocytic processes and Rosenthal fibres were reported microscopically in the white matter lesions of patients with GAN. ${ }^{28}$ In accordance with the histopathological features, recently, cerebral proton magnetic resonance spectroscopy of a patient with GAN showed findings of demyelination in the white matter, and increased amounts of choline containing compounds and myoinositol. ${ }^{17}$

The cavum septi pellucidi and vergae abnormalities were detected in the MRI of all patients, and have also been noted in other patients with GAN. ${ }^{36}$ Cavum septi pellucidi is considered to be a significant marker of aberrant brain development and is rarely seen in normal individuals (2.4\%), whereas the cavum vergae deformity occurs with the same frequency in both normal and retarded populations. ${ }^{38}$ Cavum septi pellucidi results from the failure of two primordial 
leaflets of the septum to fuse. Although its clinical importance is not known, an increased frequency of cavum septi pellucidum was found in another white matter disorder-megalencephaly and leucodystrophy with a mild clinical course. ${ }^{39}$

\section{GAN mutations}

In agreement with the known consanguinity of the parents, all affected children were homozygous for the mutation. We have already reported the R293X mutation found in family 1 in another Turkish family, where the patient was a compound heterozygote together with a C570Y mutation. ${ }^{14}$ The R293X mutation is predicted to truncate a part of the first kelch domain and five other kelch domains. In an expression study, the R293X mutation was shown to cause loss of interaction between gigaxonin and MAPIB-LC. ${ }^{22}$ Because this mutation alters a CG dinucleotide, which are known to be mutational hot spots by transition of methylated $\mathrm{C}$ to $\mathrm{T}$, the nucleotide change in both families may represent independent recurrent mutational events. However, patients in both families shared the same homozygous alleles for markers LC5 and LC6 (data not shown). According to the complete sequence of the gene from the human genome working draft, LC5 is an intragenic marker located between exons 5 and 6. Therefore, it is equally possible that the multiple occurrence of the R293X mutation in Turkey is the consequence of a common founder event.

Patients from families 2 and 3 share the same mutation, which is a splice mutation of exon $9(1502+1 G>T)$. Skipping of this exon is predicted to result in an in frame transcript missing most of the sequence encoding the fifth kelch repeat. The two families originate from nearby towns in southeastern Turkey and share a common haplotype over three consecutive markers, including the intragenic marker (LC8, LC6, and LC5). Our results suggest that the $1502+1 \mathrm{G}>\mathrm{T}$ mutation in both families derives from a common founder. The disease haplotypes of the two families also share alleles over more distal markers (LC1, LC3, and LC2), but are divergent at the intermediate marker D16S3098. This divergence may be the consequence of mutational instability at the Dl6S3098 marker locus, or less probably may reflect the occurrence of an ancestral recombination immediately distal to the GAN gene. The results presented here indicate that an initial screen for mutations in exons 5 and 9 of Turkish patients with GAN may be an efficient strategy.

Both mutations are predicted to have serious affects on the structure of gigaxonin. The associated phenotypes of the two mutations in the homozygous state were similar, except for the milder involvement of the white matter in one of the patients with the $1502+1 G>$ T mutation. Similar widespread white matter changes with brainstem and cerebellar atrophy were also seen in the cranial MRI of a patient with GAN who had a homozygous A to $\mathrm{G}$ transition in the splice donor site of intron 3, resulting in abnormally spliced gigaxonin. ${ }^{17}$ More cases are needed to make a comparison between the mutation type and CNS involvement in GAN.

Recently, using EMG, Kuhlenbäumer et al found subclinical signs of peripheral neuropathy in a heterozygous parent carrying a nonsense mutation (R201X), whereas another parent with a missense mutation (I423T) had a normal EMG. ${ }^{15}$ In our study, EMG revealed mild axonal neuropathy in the carrier of the R293X mutation but not in the carrier of $1502+1 \mathrm{G}>\mathrm{T}$. This result may relate to the more severe gigaxonin truncation caused by the R293X mutation compared with the $1502+1 G>$ T mutation. Similarly, the truncation of a part of the BTB domain and six kelch domains by the R201X mutation described by Kuhlenbäumer and colleagues $^{15}$ was sufficient to cause subclinical neuropathy in the heterozygous parent.
In conclusion, we screened the GAN gene for mutations in three Turkish families concordant for linkage to the GAN locus and identified two null mutations: the R293X mutation, previously reported in a small Turkish family, and the $1502+1 G>T$ mutation. Both mutations were associated with white matter abnormalities in cranial MRI. No major intrafamilial or interfamilial clinical variability was present among the patients carrying these two mutations. We also found subclinical neuropathy in a heterozygous carrier of the R293X mutation. These mutations leading to the truncation of gigaxonin caused a severe clinical phenotype in our patients. However, the association of severe mutations with milder phenotypes suggests the lack of a correlation between the genotype and the clinical phenotype in GAN. ${ }^{18}$ Further studies on the expression and function of gigaxonin may provide a better understanding of the pathophysiological mechanism of this disease.

\section{ACKNOWLEDGEMENTS}

We would like to thank the patients and family members who participated in this study. This work was supported in part by funds from the Institut National de la Recherche Médicale (INSERM), the Centre National de la Recherche Scientifique (CNRS) and the Hôpitaux Universitaires de Strasbourg (HUS), and the Association Française contre les Myopathies (AFM) (PB and MK). PB was supported by a fellowship from AFM.

\section{Authors' affiliations}

E Demir, S Aysun, M Topcu, $\mathrm{H}$ Topaloglu, Department of Paediatric Neurology, Hacettepe University, 06100, Ankara, Turkey

P Guicheney, INSERM U 582, Institut de Myologie, Groupe Hospitalier Pitié-Salpêtrière, Paris 75651, France

P Bomont, M Koenig, Institut de Génétique et de Biologie Moléculaire et Cellulaire, CNRS/ INSERM /ULP, Illkirch CEDEX, CU de Strasbourg 67404, France

S Erdem, M Demirci, E Tan, Department of Neurology, Hacettepe University

L Cavalier, Laboratoire de Génétique Moléculaire et Chromosomique, Institut de Génétique Humaine, Institut de Biologie, Montpellier, France G Kose, Department of Paediatric Neurology, SSK Children's Hospital, 06100 Ankara, Turkey

S Muftuoglu, A N Cakar, Department of Histology, Hacettepe University Competing interests: none declared

\section{REFERENCES}

1 Berg BO, Rosenberg SH, Asbury AK. Giant axonal neuropathy. Pediatrics 1972;49:894-9.

2 Asbury AK, Gale MK, Cox SC, et al. Giant axonal neuropathy. A unique case with segmental neurofilamentous masses. Acta Neuropathol (Berl) 1972;20:237-40

3 Maia M. Pires MM Guimaraes A. Giant axonal disease: report of three cases and review of the literature. Neuropediatrics 1988;19:10-15.

4 Ouvrier RA. Giant axonal neuropathy: a review. Brain Dev 1989;1 1:207-14.

5 Boltshauser E, Bischoff A, Isler W. Giant axonal neuropathy, report of a case with normal hair. J Neurol Sci 1977;31:269-78.

6 Malandrini A, Dotti MT, Battisti C, et al. Giant axonal neuropathy with subclinical involvement of the central nervous system: a case report. I Neurol Sci 1998;158:232-5.

7 Peiffer J, Schlote W, Bischoff A, et al. Generalized giant axonal neuropathy. A filament-forming disease of neuronal, endothelial, glial and Schwann cells in a patient without kinky hair. Acta Neuropathol (Berl) 1977;40:213-18

8 Ben Hamida M, Hentati F, Ben Hamida C. Giant axonal neuropathy with inherited multisystem degeneration in a Tunisian kindred. Neurology 1990;40:245-50.

9 Prineas JR, Ouvrier RA, Wright JC, et al. Giant axonal neuropathy-a generalised disorder of cytoplasmic microfilament formation. J Neuropathol Exp Neurol 1976;35:458-70.

10 Fois A, Balestri P, Farnetani MA, et al. Giant axonal neuropathy: endocrinological and histological study. Eur J Pediatr 1985;144:274-80.

11 Ben Hamida C, Cavalier L, Belal S, et al. Homozygosity mapping of giant axonal neuropathy gene to chromosome 16q24.1. Neurogenetics 1997; 1:129-33.

12 Flanigan KM, Crawford TO, Griffin JW, et al. Localization of the giant axonal neuropathy gene to chromosome 16q24. Ann Neurol 1998;43:143-8.

13 Cavalier L, Ben Hamida C, Amouri R, et al. Giant axonal neuropathy locus refinement to a $<590 \mathrm{~kb}$ critical interval. Eur J Hum Genet 2000;8:527-34. 
14 Bomont $\mathrm{P}$, Cavalier $\mathrm{L}$, Blondeau $\mathrm{F}$, et al. The gene encoding gigaxonin, a new member of the cytoskeletal BTB/kelch repeat family, is mutated in giant axonal neuropathy. Nat Genet 2000;26:370-4

15 Kuhlenbäumer G, Young P, Oberwittler $C$, et al. Giant axonal neuropathy (GAN): case report and two novel mutations in the gigaxonin gene. Neurology 2002;58:1273-6.

16 Bomont $\mathrm{P}$, loos $\mathrm{C}$, Yalcinkaya $\mathrm{C}$, et al. Identification of seven novel mutations in the GAN gene. Hum Mutat 2003;21:446-51.

17 Brockman K, Pouwels PJW, Dechent $P$, et al. Cerebral proton magnetic resonance spectroscopy of a patient with giant axonal neuropathy. Brain Dev 2003;25:45-50.

18 Bruno C, Bertini E, Federico A, et al. Clinical and molecular findings in patients with giant axonal neuropathy (GAN). Neurology 2004:62:13-16.

19 Robinson DN, Cooley L. Drosophila kelch is an oligomeric ring canal actin organiser. J Cell Biol 1997;138:799-810.

20 Hernandez MC, Andres-Barquin PJ, Martinez S, et al. ENC-1: a novel mammalian kelch-related gene specifically expressed in the nervous system encodes an actin-binding protein. J Neurosci 1997; 17:3038-51.

21 Soltysik-Espanola M, Rogers RA, Jiang S, et al. Characterisation of mayven, a novel actin-binding protein predominantly expressed in brain. Mol Biol Cell 1999:10:2361-75.

22 Ding J, Liu J, Kowal AS, et al. Microtubule-associated protein 1B: a neuronal binding partner for gigaxonin. J Cell Biol 2002; 158:427-33.

23 Tögel M, Wiche G, Propst W. Novel features of the light chain of microtubuleassociated protein MAPIB: microtubule stabilization, self interaction, actin filament binding and regulation by the heavy chain. J Cell Biol 1998; 143:695-707

24 Bomont $\mathbf{P}$, Koenig $M$. Intermediate filament aggregation in fibroblasts of giant axonal neuropathy patients is aggravated in non dividing cells and by microtubule destabilization. Hum Mol Genet 2003;12:813-22.

25 Donaghy M, Brett EM, Ormerod IEC, et al. Giant axonal neuropathy: observations on a further patient. J Neurol Neurosurg Psychiatry 1988:51:991-4.

26 Sabatelli $M$, Bertini E, Servidei S, et al. Giant axonal neuropathy: report on a case with focal fiber loss. Acta Neuropathol (Berl) 1992;83:543-6.
27 Kretzscmar HA, Berg BO, Davis RL. Giant axonal neuropathy: a neuropathological study. Acta Neuropathol (Berl) 1987;73:138-44.

28 Thomas C, Love S, Powell HC, et al. Giant axonal neuropathy: correlation of clinical findings with postmortem neuropathology. Ann Neurol 1987;22:79-84.

29 Larbrisseau A, Jasmin G, Hausser C, et al. Generalized giant axonal neuropathy. A case with features of Fazio-Londe disease. Neuropadiatrie 1979;10:76-86.

30 Guazzi GC, Malandrini A, Gerli R, et al. Giant axonal neuropathy in 2 siblings: a generalised disorder of intermediate filaments. Eur Neurol 1991;31:50-6

31 Igisu $H$, Ohta $M$, Tabira $T$, et al. Giant axonal neuropathy. A clinic entity affecting the central as well as the peripheral nervous system. Neurology 1975:25:717-21

32 Takebe Y, Koide N, Takahashi G. Giant axonal neuropathy: report of two siblings with endocrinologic and histological studies. Neuropediatrics $1981 ; 12: 392-404$

33 Lampl Y, Eshel Y, Ben David E, et al. Giant axonal neuropathy with predominant central nervous system manifestations. Dev Med Child Neurol 1992;34:164-81.

34 Zemmouri R, Azzedine H, Assami S, et al. Charcot-Marie-Tooth 2-like presentation of an Algerian family with giant axonal neuropathy. Neuromuscul Disord 2000;10:592-8.

35 Richen P. Tandan R. Giant axonal neuropathy: progressive clinical and radiologic CNS involvement. Neurology 1992;42:2220-2.

36 Trebier-Held S, Budjarjo-Welim, Riemann D, et al. Giant axonal neuropathy: a generalised disorder of intermediate filaments with longitudinal grooves in the hair. Neuropediatrics 1994;25:89-93.

37 Stollhoff K, Albani M, Goebel HH. Giant axonal neuropathy and leukodystrophy. Pediatr Neurol 1991;7:69-71.

38 Bodensteiner JB, Schaefer GB, Craft JM. Cavum septi pellicidi and cavum vergae in normal and developmentally delayed populations. J Child Neurol 1998;13:120-1.

39 Topcu M, Saatci I, Topcuoglu MA, et al. Megalencephaly and leukodystrophy with mild clinical course: a report on 12 new cases. Brain Dev 1998;20: 142-53 